\title{
Six Algorithms for Frequency Offset Estimation in OFDM Systems
}

\author{
Ahmed M. Alaa \\ ECEE Dept., Faculty of Engineering, Cairo University, Gizah , Egypt \\ E-mail: aalaa@eece.cu.edu.eg
}

\begin{abstract}
Generally, wireless systems suffer from Carrier Frequency Offsets (CFO), which results either from the receiver's oscillator's impairments or Doppler shifts. The frequency offset between transmitter and receiver local oscillator is a main drawback of OFDM systems. A frequency drift in the receiver's oscillator would result in a frequency offset in the received signal, this offset consists of two parts: a Fine offset, as a fraction of OFDM subcarrier spacing, and a Coarse offset that is equal to an integer multiple of the subcarrier spacing. Coarse offsets results in two problems; firstly, the modulation symbols are located on the wrong frequency bins, secondly, the pilots used for channel estimation are misplaced leading to a faulty channel estimate and severe signal distortion by the Equalizer. Fine Offsets result in a loss of orthogonality and thus ICI (Inter-Carrier Interference), as the frequency bin of a certain modulation symbol would contain some information about the neighboring symbol bin.
\end{abstract}

Index Terms - Orthogonal Frequency Division Multiplexing (OFDM), Carrier Frequency Offset (CFO), Frequency Offset Estimation, Frequency Synchronization, Baseband Processing

\section{Introduction}

We consider the problem of oscillator mismatch between the transmitter and receiver as a part of wireless transmission impairments. The oscillator's mismatch can occur via a frequency drift in the receiver, transmitter or both. We will refer the frequency offset to the receiver's oscillator.

The receiver is assumed to have a frequency drift of $\Delta f$, in spite that specifications are imposed on the crystal oscillators' accuracy, the high sensitivity of the OFDM systems to frequency errors makes seeking an algorithm for frequency offset estimation inevitable [1]. As stated before, the frequency offset can be decomposed into two parts: A fine offset and a coarse offset. Coarse offsets result in two problems; improper modulation symbols mapping to frequency bins and a catastrophic degradation in the quality of channel estimation as pilot locations are completely jumbled [2]. Fine Offsets result in a loss of orthogonality and thus ICI (Inter-
Carrier Interference), as the frequency bin of a certain modulation symbol would contain some information about the neighboring symbol bin with the actual modulation symbol has its amplitude decreased [3]. The paper is organized as follows: in section II we demonstrate the effect of frequency offset on OFDM signals. Next, in section III, we propose six offset estimation algorithms. Finally, we draw our conclusion in section IV.

\section{Frequency Offset Effects}

In this section, we discuss in details the effect of the frequency offset on the OFDM scheme. Next, we propose six novel algorithms for frequency offset estimation, exploiting the unique properties of the OFDM symbols' structure and the receiver's knowledge of the time domain pilots (known symbols transmitted by the receiver).

OFDM sensitivity to frequency offsets arise from being a Multi-carrier modulation scheme. The fact that specific frequency bins carry independent information makes altering the frequency bins locations intolerable [4][5]. A transmitted baseband signal $x(t)$ would be perceived by the receiver with an oscillator mismatch as $x(t) \mathrm{e}^{\mathrm{j} 2 \pi \Delta f \mathrm{t}}$. Recall the fourier transform property:

$$
x(t) \mathrm{e}^{\mathrm{j} 2 \pi \Delta f \mathrm{t}} \leftrightarrow X(f-\Delta f)
$$

Thus, a multiplication by a complex exponential in the time domain causes a frequency shift in the frequency domain. Because the DFT (Discrete Fourier Transform) has a circular nature, we note that this shift comes to be a circular shift of the signal's spectrum $X(f)$.

For instance, if we have a 6 subcarrier OFDM system, a Coarse offset of 1 subcarrier spacing translates subcarrier 6 to the frequency bin related to subcarrier 1 , and shifts all other subcarriers by 1 bin. Assume the receiver tries to decode the incoming OFDM symbol, how can it determine which bin maps to which subcarrier? Another important problem is that if subcarriers 2, 4 and 6 where pilot tones, now they are replaced by different subcarriers and the pilot values are not known anymore; any pilot aided Channel estimation scheme will fail under this scenario. Thus estimation of the Coarse offset is mandatory. In Addition, the Fine 
Offset shows a loss of orthogonality as each frequency bin contains contributions from neighboring subcarriers and a loss in the amplitude of the desired subcarrier is noted.

From the above discussion, it is apparent that an algorithm for frequency offset estimation is mandatory for proper system operation. We propose six different algorithms for Offset estimation; the algorithms are given the following names:

1. The Spectral-Correlation Algorithm (AWGN channel, Joint Fine and Coarse offset detection)

2. The Linear Phase Smoothening Algorithm (AWGN channel, Joint Fine and Coarse offset detection)

3. Blind Cyclic-prefix based Frequency Offset estimation Algorithm (Fading channel, Fine offset detection) and Large Span Cyclic-prefix based Algorithm

4. Sample-level frequency offset estimation Algorithm (Ideal channel, Joint Fine and Coarse offset detection)

5. Frequency Offset estimation by Phase difference Spectral decomposition (AWGN channel, Joint Fine and Coarse offset detection)

6. Iterative Cyclic-prefix based algorithm (Fading channel, Fine and Coarse offset detection)

\section{Frequency Offset Estimation Algorithms}

In this section we discuss the six proposed algorithms for estimating the frequency offset in OFDM:

\subsection{The Spectral Correlation Algorithm}

The first proposal relies on the utilization of time domain pilots (a known symbol by the receiver). Because the time domain structure of the pilots is known, the receiver can deduce the frequency domain structure of this specific OFDM symbol.

Because the effect of the frequency offset is a shift in the frequency domain, then the received pilot symbol has a spectrum that is a circular shift of a frequency domain structure that we already know. Let the pilot symbol's frequency domain structure be denoted by $T(f)$, and the received symbol's DFT as $\widetilde{T}(f)$, we define a the cross-correlation function as (E[.] is the expected value:

$$
\mathrm{C}(g)=\mathrm{E}\left[T^{*}(f) \widetilde{T}(f+g)\right]
$$

Because the cross correlation function measures the similarity between a signal and a shifted version of it across different shift, it is logical that function $\mathrm{C}(g)$ will have a maximum value at exactly $g=\Delta f$. Thus our estimate can be formulated as:

$$
\Delta \hat{f}=\max _{g} C(g)
$$

This estimate bundles both the Coarse and Fine offsets together with no discrimination. The Algorithm provides satisfactory performance in AWGN.

An important issue regarding the spectral correlation algorithm is the finest frequency offset it can detect. Because $T(f)$ and $\widetilde{T}(f)$ are obtained via N-point FFT, thus we have one sample per subcarrier, and the Algorithm cannot detect the fine component with $\mathrm{N}$ point FFT, only Coarse component is detected.

A minor modification to the Algorithm above is to obtain $T(f)$ and $\widetilde{T}(f)$ via $\mathrm{N}_{\text {extra-point FFT. Thus, if we }}$ want to detect frequency offsets that are as fine as 0.1 subcarriers, then $\mathrm{N}_{\text {extra }}=10 \mathrm{~N}$, where $\mathrm{N}$ is the OFDM transmission mode.

The Spectral Correlation algorithm is very robust when applied to AWGN channels. The fact that noise components in the frequency domain have no or little effect on the degree of similarity between the signal's DFT and its shifted version means that the estimation quality will be SNR (Signal-to-Noise Ratio) independent. Fig. 1 shows the resilience of the estimate over low and high SNR channels.

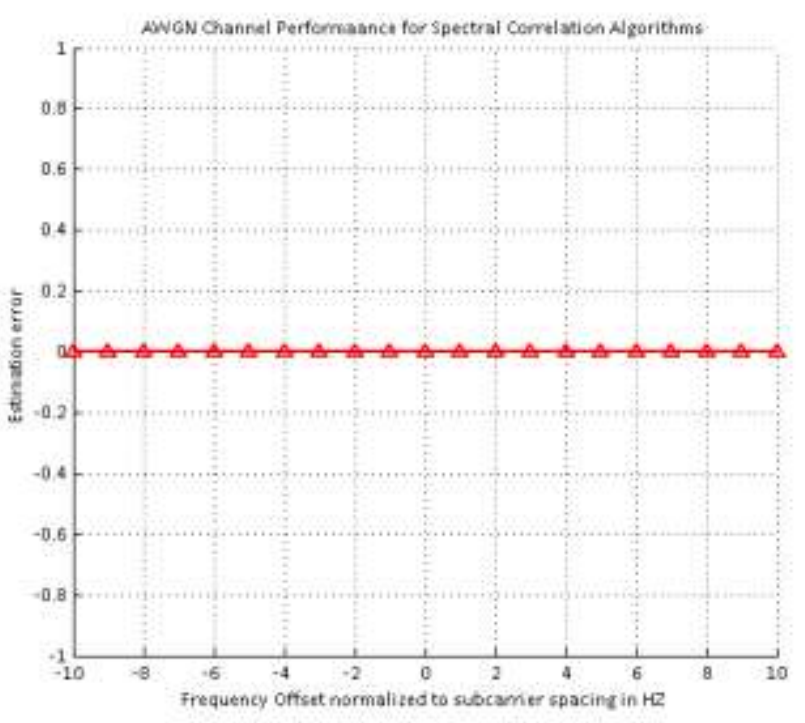

Fig. 1: Estimation error versus subcarrier spacing in AWGN

However, a severe challenge arises when dealing with fading channels. If the channel exhibits very large values for the delay spread and frequency selective behaviour become inevitable, the received signal has a frequency domain structure of $T(f) . H(f)$, where $H(f)$ is the channel response. The performance becomes catastrophically degraded in frequency selective channels and the Algorithm is no more reliable. In spite of the fact that flat fading channels have no impact on the Algorithm's operation, an OFDM system's receiver will rarely face a flat channel. 


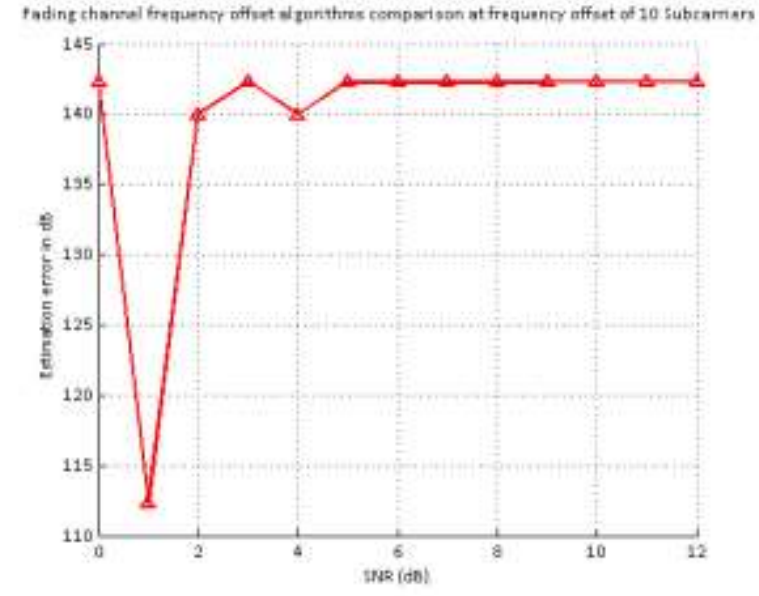

Fig. 2: The estimation error over a range of different SNRs in a frequency selective channel, the error is as high as $140 \mathrm{~dB}$, and the algorithm becomes unreliable

\subsection{Linear Phase Smoothening Algorithm}

The previous method was a frequency domain technique that exploited the knowledge of the frequency domain structure of the time domain pilot. The time domain structure of the pilot channel is of course known as well. The Linear-Phase smoothening Algorithm exploits this knowledge to estimate the frequency offset in the time domain. Assume that the received time domain pilot symbol and the known one are denoted by $S(t)$ and $S(t)$ respectively. Each of the two signals is composed of $\mathrm{N}$ chips. Assuming an ideal channel where no fading occurs and no addition of noise, then:

$$
S(t)=S(t) \cdot e^{j 2 \pi \Delta f t}
$$

The phase difference between the two signals per chip, assuming that the phase of $x$ is $\arg (x)$ :

$$
\begin{aligned}
\arg (S(t))-\arg (S(t)) & = \\
\arg \left(S(t) \cdot e^{j 2 \pi \Delta f t}\right) & -\arg (S(t)) \bmod 2 \pi \\
& =2 \pi \Delta \mathrm{ft}
\end{aligned}
$$

Thus, when the phase difference is plotted with time, it is a straight line of a slope of $2 \pi \Delta \mathrm{f}$, if we managed to calculate this slope, we will be able to detect the frequency offset, and hence the name, linear phase smoothening. The effect of noise on the phase difference is not catastrophic, because a noise sample with low amplitudes in the real and imaginary components is added to a received chip will not change its phase too much as the real and imaginary components of the signal's chip is expected to dominate, thus noise causes slight fluctuations around a local mean value, this mean value corresponds to the correct frequency offset. The fine and the coarse offsets are estimated jointly with no discrimination, due to the fact that they together decide the slope of the phase difference.

The effect of fading is again catastrophic. Fading can be modeled in the time domain as a complex random variable multiplied by the signal, thus the phase of channel gain in the time domain is directly added to the phase of a chip, each frequency component (sinusoidal) views a different channel gain in a frequency selective channel, and thus, the phase difference between the received and the known pilot symbols' chips cannot show a clear slope that we can track, so the method offers an unreliable performance.

If the frequency offset has a quite high value (more than one subcarrier spacing), then the phase difference may reach $2 \pi$ several times within the symbol duration and we have a saw tooth signal with many slopes each of them corresponds to the frequency offset. To calculate the slope, we set two boundary values $\mathrm{N}_{1}$ and $\mathrm{N}_{2}$ as chip indexes. The slope is calculated between them, then $\mathrm{N}_{2}$ is shifted by a certain value and the slope is re-calculated. $\mathrm{N}_{2}$ is shifted again and the process is repeated iteratively until the slope values fixes.

Because noise causes fluctuations about a mean desired value, the calculated slope may be faulty and the iterations may never converge as $\mathrm{N}_{2}$ may probably coincide with chips of low SNR. Thus, we apply the phase difference saw tooth signal to a low pass filter (moving average filter), this calculates a time domain average value or removes most of the white noise power in the frequency domain. The results are quite unstable for AWGN channels.

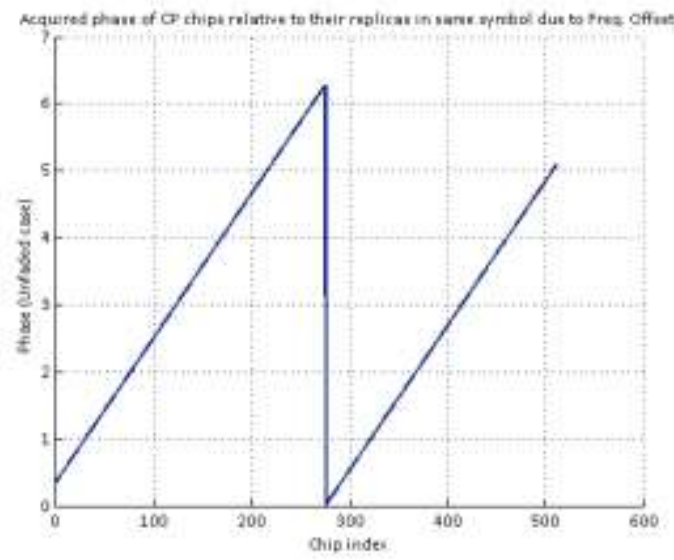

Fig. 3: The Phase difference signal over an ideal channel, the slope of the saw tooth denotes the frequency offset

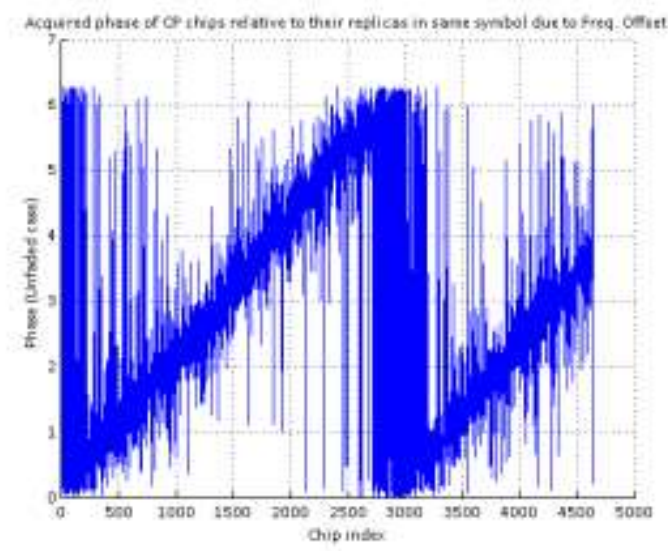

Fig. 4: The effect of noise on the phase difference, fluctuations around the straight line with the targeted slope can disturb the slope detection process 


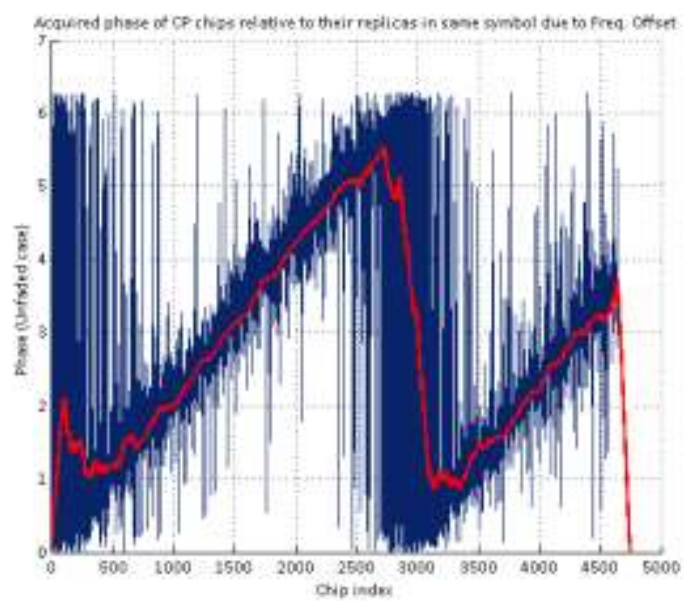

Fig. 5: Smoothening is applied by a moving average low pass filter to get a reliable saw tooth function for slope detection. The dark curve is the noise affected phase difference and the red curve is the filtered one

\subsection{Blind Cyclic Prefix Algorithm}

The previous methods were unreliable for fading channels, which is the class of channels usually encountered by mobile receivers. The third algorithm utilizes the cyclic nature of the time domain structure of an OFDM symbol to estimate the frequency offset and eliminate the fading effects in a smart way. This technique doesn't use a predefined symbol (time domain pilot) and hence the name 'Blind Cyclic-prefix based algorithm'. Assume an OFDM symbol $S[n]$ is received and it consists of $\mathrm{N}+\mathrm{N}_{\mathrm{cp}}$ chips, with $\mathrm{N}$ useful chips and $\mathrm{N}_{\mathrm{cp}}$ cyclic prefix chips. Exploiting the cyclic nature of the OFDM symbol we know that the first $\mathrm{N}_{\mathrm{cp}}$ chips are identical to the last $\mathrm{N}_{\mathrm{cp}}$ chips. The phase between corresponding chips within the first and last $\mathrm{N}_{\mathrm{cp}}$ sized windows identifies the frequency offset. The power of this method resides in the presence of $\mathrm{N}_{\mathrm{cp}}$ estimates of the frequency offset (phase difference between every two corresponding chips in the first and last $\mathrm{N}_{\mathrm{cp}}$ set of chips), and thus a representative value for them would be far more precise than the estimates obtained by the previous algorithms due to the time diversified nature of the $\mathrm{N}_{\mathrm{cp}}$ estimates.

For $\mathrm{S}[\mathrm{n}]$ being the received time domain OFDM symbol, $\mathrm{T}_{\mathrm{c}}$ the chip duration and $\mathrm{T}_{\mathrm{u}}$ the useful period $\left(\mathrm{NT}_{\mathrm{c}}\right)$ we can find $\mathrm{N}_{\mathrm{cp}}$ estimates for the frequency offset as:

$$
\Delta \tilde{f}_{\mathrm{k}}=\frac{(\arg (\mathrm{s}[\mathrm{k}+\mathrm{N}])-\arg (\mathrm{s}[\mathrm{k}])) \bmod 2 \pi}{2 \pi \mathrm{Tu}},
$$

Thus, we have a vector of $\mathrm{N}_{\mathrm{cp}}$ estimates $\tilde{\Delta} \tilde{\boldsymbol{f}}$, to get a representative estimate value for them we obtain their average as:

$$
\Delta \tilde{f}=\frac{1}{N c p} \sum_{i=0}^{N c p-1} \Delta \tilde{f}_{\mathrm{i}}
$$

Elimination of fading and noise is done by this averaging; the estimated value obtained is very accurate To further study the effect of averaging on fading assume we receive a faded signal $S[n] C[n]$, where $C[n]$ is the channel gain in the time domain at the OFDM symbol chips, thus:

$$
\begin{aligned}
& \Delta \tilde{f}_{\mathrm{k}}= \\
& \frac{(\arg (\mathrm{s}[\mathrm{k}+\mathrm{N}] \mathrm{C}[\mathrm{k}+\mathrm{N}])-\arg (\mathrm{s}[\mathrm{k}] \mathrm{C}[\mathrm{k}])) \bmod 2 \pi}{2 \pi \mathrm{Tu}}, \\
& \mathrm{k}=0,1, \ldots \ldots \mathrm{N}_{\mathrm{cp}}-1
\end{aligned}
$$

where $\mathrm{S}[\mathrm{n}]$ is a single tone. Let the vector $\underline{\Delta} \tilde{f}$ in this case be:

$$
\begin{aligned}
\underline{\Delta \boldsymbol{f}}= & {\left[\Delta f+\varphi_{1}-\alpha_{1}, \Delta f+\varphi_{2}-\alpha_{2},\right.} \\
& \left.\Delta f+\varphi_{3}-\alpha_{3}, \ldots \ldots, \Delta f+\varphi_{\mathrm{Ncp}}-\alpha_{\mathrm{Ncp}}\right]
\end{aligned}
$$

where $\varphi_{k}$ and $\alpha_{k}$ are the phases of the channel gain at the $\mathrm{k}^{\text {th }}$ chip in addition to the effect of noise in the first $\mathrm{N}_{\mathrm{cp}}$ and the last $\mathrm{N}_{\mathrm{cp}}$ windows, by averaging as equation (7) implies:

$$
\Delta \tilde{f}=\Delta f+\frac{1}{N c p} \sum_{i=0}^{N c p-1} \varphi \mathrm{i}-\alpha \mathrm{i}
$$

Assuming that both $\varphi_{\mathrm{i}}$ and $\alpha_{\mathrm{i}}$ have the same probability density functions with a mean of $\boldsymbol{\mu}$, if $\mathrm{N}_{\mathrm{cp}}$ is large enough, we find that:

$$
\begin{aligned}
& \Delta \tilde{f}=\Delta f+\frac{1}{N c p} \sum_{i=0}^{N c p-1} \varphi \mathrm{i}-\frac{1}{N c p} \\
& \sum_{i=0}^{N c p-1} \alpha \mathrm{i} \cong \Delta f+\mu-\mu=\Delta f
\end{aligned}
$$

In the previous equations, we obtained a vector of estimates $\underline{\Delta} \tilde{f}$ based on every sample (chip) in the cyclic prefix. Averaging on these estimates would eliminate the effect of noise, but one might think of the role of fading in distorting the offset estimates as being eliminated by subtracting the corresponding chips of data and cyclic prefix, because each chip is a linear combination of sinusoids at a certain time instant, and each sinusoid is affected by a different channel gain, when subtracting the two chips, the phase acquired by the channel gain will be eliminated. This is based on the assumption that the channel is constant over an OFDM symbol.

In fact, this is only true for a stationary receiver or a time invariant channel, however, when the receiver moves even at low speeds, we cannot guarantee that all the frequency components have their gains constant over the entire OFDM symbol because the coherence time is a statistical variable. Some frequency components in the data chips may have different gain from that perceived by the cyclic prefix chips, and the phase difference is not zero. 
We note that use of phase difference to detect the frequency offset means that ambiguity occurs in our estimation. Our estimate can only identify the smallest offset frequency satisfying the phase condition between $\mathrm{S}[\mathrm{k}]$ and $\mathrm{S}[\mathrm{k}+\mathrm{N}]$ chips. However, other offset frequencies with extra integer multiples of $2 \pi$ will not be detectable. This is analogous to the phase condition that identifies existing modes in a cavity resonator. Assume an ideal channel and the phase difference between two samples in $\mathrm{S}[\mathrm{k}]$ and $\mathrm{S}[\mathrm{k}+\mathrm{N}]$ is $\omega_{\mathrm{o}}$, thus:

$\Delta \omega \mathrm{Tu}=\omega_{\mathrm{o}}$ yields same estimate as

$\Delta \omega \mathrm{Tu}=\omega_{\mathrm{o}}+\mathrm{m} 2 \pi, \mathrm{m}=0,1,2, \ldots$

The estimated frequency offset becomes $\Delta \omega=\frac{\omega o}{\text { Tu. }}$, the maximum correctly estimated offset is at $\Delta \omega \mathrm{Tu}=2 \pi$ and is given by:

$$
\Delta \omega=\frac{2 \pi}{T u}
$$

If the system bandwidth is $W$, and $\mathrm{Tu}=\frac{N}{W}$, and the subcarrier spacing $f_{s}=\frac{W}{N}=\frac{1}{T u}$, the maximum detectable offset is:

$$
\Delta \omega \leq \frac{2 \pi}{T u}=2 \pi f s=\omega_{\mathrm{s}}
$$

Thus, the cyclic prefix based method can only estimate the fine component; the coarse component goes transparently undetectable. A method to estimate the coarse component separately (Large Span CyclicPrefix based Algorithm) was developed, it obtains the phase difference signal between the time domain pilot and the received signal, and counts the number of humps in the saw tooth signal that corresponds to the number of subcarriers in the coarse offset component. However, the use of the phase difference signal limits the algorithm's capabilities to combat fading as it will suffer from the same susceptibilities of the previous methods, and the ability to eliminate fading (which is the source of attractiveness for this algorithm) vanishes.

Estimation of negative frequency offsets is straightforward, we can either detect the fine offsets from $[0,1]$ subcarrier spacing, or shift the phase ambiguity to be an even function of the frequency offset; if the detected offset is more than 0.5 of a spacing, we subtract 1 from it.

\subsection{Sample Level Based Algorithm}

This algorithm only modifies the previous algorithm trying to extend its span of detectable frequency offsets. Recall that the maximum detectable frequency offset in the Cyclic-prefix based algorithm was given by $\Delta \omega=$ $2 \pi$

$\overline{T u}$, thus, we can increase $\Delta \omega$ by decreasing the period between the chips that we compare their phases. The largest achievable value for $\Delta \omega$ is when replacing $T_{u}$ by $\mathrm{T}_{\mathrm{c}}$ (chip duration). Assume we have the received time domain pilot as S[n] and the actual time domain pilot as $\mathrm{S}[\mathrm{n}]$, then algorithm tries to get the phase difference between neighboring samples and compares them with phase difference in neighboring samples in the actual correct time domain pilot symbol, the usage of neighboring samples makes the phase condition harder to satisfy by many offsets and increases the span of $\Delta \omega$.

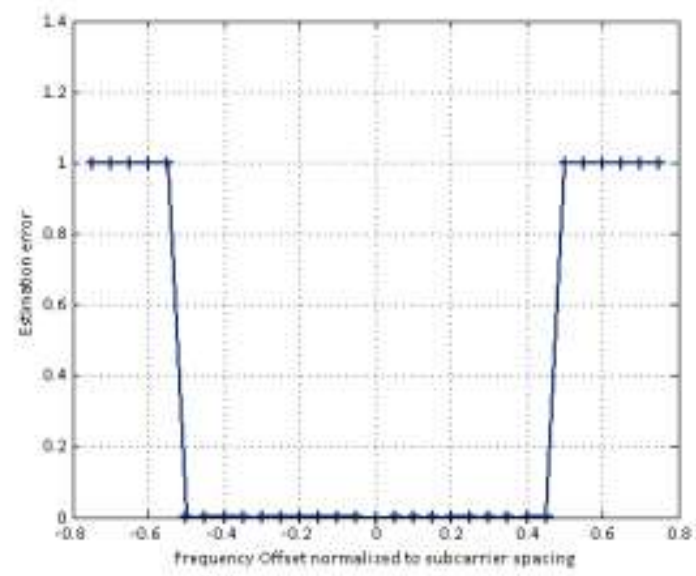

Fig. 6: Ideal channel estimation error, note that we can detect offsets in the range [-0.5. 0.5$]$

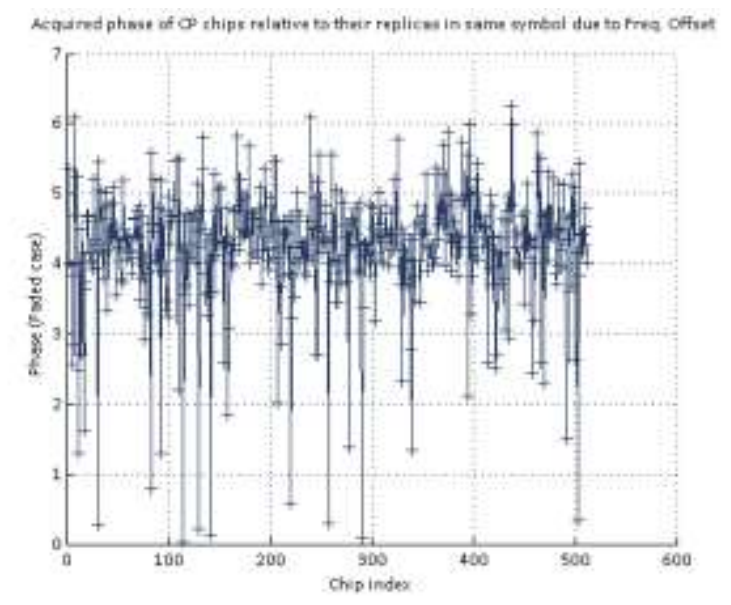

Fig. 7: The vector of estimates affected by fading and noise. Averaging obtains a precise estimate for the frequency offset

The Algorithm is formulated as follows:

$$
\begin{aligned}
& \tilde{f}_{\mathrm{k} 1}=(\arg (\tilde{S}[\mathrm{n}])-\arg (\tilde{S}[\mathrm{~m}])), \\
& \mathrm{n}=2,4,6, \ldots \mathrm{Ncp} \text { and } \mathrm{m}=1,3,5, \ldots ., \mathrm{Ncp}-1 \\
& \tilde{f}_{\mathrm{k} 2}(\arg (\mathrm{S}[\mathrm{n}])-\arg (\mathrm{S}[\mathrm{m}])), \\
& \mathrm{n}=2,4,6, \ldots \mathrm{Ncp} \text { and } \mathrm{m}=1,3,5, \ldots ., \mathrm{Ncp}-1
\end{aligned}
$$




$$
\begin{aligned}
& \Delta \tilde{f}_{\mathrm{k} 3}=(\arg (\tilde{S}[\mathrm{n}+\mathrm{N}])-\arg (\tilde{S}[\mathrm{~m}+\mathrm{N}])) \\
& \Delta \tilde{f}_{\mathrm{k} 4}(\arg (\mathrm{s}[\mathrm{n}+\mathrm{N}])-\arg (\mathrm{s}[\mathrm{m}+\mathrm{N}])) \\
& \mathrm{X}=\left(\Delta \tilde{f}_{\mathrm{k} 1}-\Delta \tilde{f}_{\mathrm{k} 2}\right) \bmod 2 \pi \\
& \mathrm{Y}=\left(\Delta \tilde{f}_{\mathrm{k} 3-\Delta} \tilde{f}_{\mathrm{k} 4}\right) \bmod 2 \pi \\
& \tilde{f}=\frac{1}{\operatorname{TcN} N c p} \sum\left(\frac{X+Y}{2}\right) \bmod 2 \pi
\end{aligned}
$$

The algorithm works perfectly on the ideal channel, however, it is extremely numerically sensitive, because the phase calculates is very small valued the approximation we made in equation (11) is not valid anymore and the difference between the channel gain phases averages dominates. Thus, this method is not suitable either for AWGN or fading channels.

\subsection{Phase Spectral Decomposition Algorithm}

Recall the saw tooth phase difference signal obtained for the linear phase smoothening algorithm, this signal appears (for a large frequency offset) to be a quasi periodic signal. Thus, its Fourier transform (DFT) will result perfect impulses with the largest harmonic being the fundamental period of the saw tooth signal. Note that if the slope of a tooth is $2 \pi \Delta \mathrm{f}$, and the height is $2 \pi$ (phase wrapping), then the period has to be $\frac{1}{\Delta f}$. By calculating the DFT of the phase difference saw tooth signal after removing its DC power, the largest spectral component is located at the fundamental frequency bin which is the same as the frequency offset. This method, like other frequency domain methods, is resilient to noise but fails for frequency selective fading.
This algorithm has three main drawbacks:

- The frequency selective fading distorts the phase spectral components

- The fact that the signal is short-term periodic means that its spectrum will be spread due to spectral leakage, and identifying the point for maximum is difficult

- Can’t work for negative offsets as we only observe the signals periodicity

\subsection{Iterative Cyclic Prefix Based Algorithm}

We conclude that the Cyclic-prefix based algorithm is the most resistant to fading effects but is limited to fine estimates only. The last proposed algorithm is an iterative algorithm that obtains the fine estimate, divides by a complex exponential with the fine offset as its argument, and then it estimates the channel and equalizes the OFDM symbol. Every iteration, we check the retrieved bits, if a certain threshold of Bit errors is attained we exit, else, we divide by a complex exponential whose argument is the complete subcarrier spacing (coarse part).

Assume a generic fine and coarse offset error defined as:

$$
\Delta \omega=\omega_{\mathrm{o}}+\mathrm{m} \frac{2 \pi}{T u}
$$

where $\omega_{0}$ is the fine part and $\mathrm{m} \frac{2 \pi}{T u}$ is the coarse part. We first estimate $\omega_{0}$ using the cyclic prefix algorithm and eliminate it, the residual offset becomes $\mathrm{m} \frac{2 \pi}{T u}$, we iteratively eliminate $\frac{2 \pi}{T u}$ offset error per iteration until correctly decoding the time domain pilot bits.

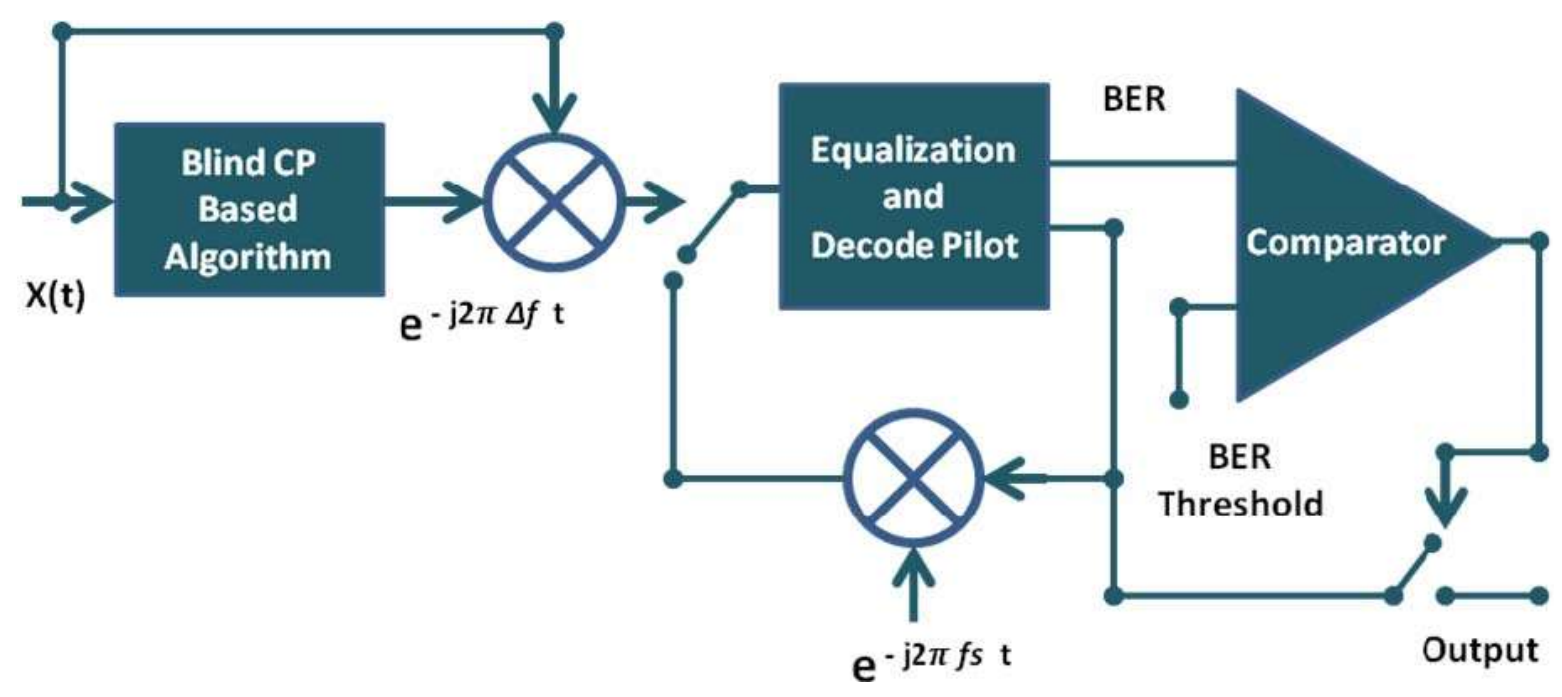

Fig. 8: Block diagram for the iterative cyclic prefix based algorithm 
This algorithm offers reasonable performance across fading channels; however its performance is highly determined by the quality of the channel estimator and equalizer. If channel estimation is quite accurate, the algorithm is reliable. The overall performance comparison between all the proposed algorithms is shown in fig. 9 .

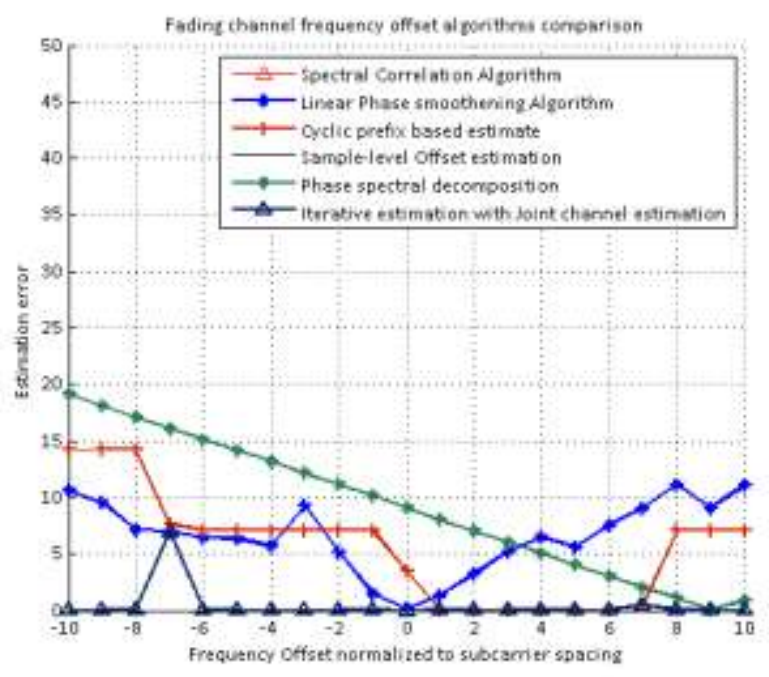

Fig. 9: Residual error versus frequency offset for various algorithms

\section{Conclusions}

The effect of frequency offsets on the OFDM system was presented, and was found to have obvious impact on the system performance. Six Algorithms for estimating the frequency offset were proposed. The Spectral Correlation Algorithm was presented, exploiting the frequency domain correlation properties of the received OFDM symbol pilot, but found to be inefficient in frequency selective fading. The Linear phase smoothening algorithm was proposed to exploit the pilots in the time domain, but not robust for fading channels. The Blind Cyclic prefix algorithm utilizes the cyclic nature of the OFDM symbol to eliminate the effect of fading and provides the most reliable results.

\section{References}

[1] T. Pollet, M. van Bladel, and M. Moeneclaey, 'BER sensitivity of OFDM systems to carrier frequency offset and Wiener phase noise', IEEE Transactions on Communications, vol. 43, no. 2/3/4, pp. 191-193, February/March/April 1995.

[2] Shiou-Hong Chen, Way-Hong He, Hou-Shin Chen, Yumin Lee, 'Mode Detection, Synchronization, and Channel Estimation for DVB-T OFDM Receiver', GLOBECOM 2003, IEEE, 2003

[3] J.A.C. Bingham, 'Multicarrier modulation for data transmission: An idea whose time has come', IEEE
Communications Magazine, vol. 28, no. 5, pp. 5-14, May 1990.

[4] Z. Cvetkovic, V. Tarokh, S. Yoon, "On frequency offset estimation in OFDM", IEEE Transactions on Wireless Communications, Vol. 12, no. 3, pp. 1062 - 1072, March 2013.

[5] D. Zhang, L. Yu, P. Zhou, and Q. Huang, “A High Performance Frequency Offset Estimation Method for OFDM", Third International Conference on Intelligent System Design and Engineering Applications (ISDEA), Hong Kong, pp. 73 - 76, Jan. 2013

[6] X. Chai, Z. Zhang, X. Zhang , and K. Long, "Efficient carrier frequency offset estimation for OFDM using periodic training sequences", Wireless and Optical Communication Conference (WOCC), Chongqing, 16-18 May 2013

[7] L. Danping, Li Yongzhao, H. Zhang , L. J. Cimini, "Integer Frequency Offset Estimation for OFDM Systems With Residual Timing Offset Over Frequency Selective Fading Channels", IEEE Transactions on Vehicular Technology, Vol. 61 , No. 6, July 2012

\section{Author's Profiles}

Ahmed M. Alaa is graduated from the department of Communications and Computer Engineering, Cairo University, Egypt in 2011. His research interests include: Wireless communications, OFDM based systems (Channel estimation, Frequency Offset estimation, Time synchronization,...etc), Mobile TV standards and Signal processing for Mobile Communications.

How to cite this paper: Ahmed M. Alaa,"Six Algorithms for Frequency Offset Estimation in OFDM Systems", International Journal of Information Technology and Computer Science(IJITCS), vol.6, no.5, pp.36-42, 2014. DOI: 10.5815/ijitcs.2014.05.05 